\title{
Influence of Viscous Dissipation on Free Convection in a Non-Darcy Porous Medium Saturated with Nanofluid in the Presence of Magnetic Field
}

\author{
Ch. RamReddy, ${ }^{1, *}$, P.V.S.N. Murthy ${ }^{2}$, A.J. Chamkha ${ }^{3}$ and A.M. Rashad ${ }^{4,5}$ \\ ${ }^{1}$ Department of Mathematics, National Institute of Technology, Rourkela-769008, Odisha, India \\ ${ }^{2}$ Department of Mathematics, Indian Institute of Technology, Kharagpur-721 302, India \\ ${ }^{3}$ Manufacturing Engineering Department, Public Authority for Applied Education and Training, Shuweikh, 70654, Kuwait \\ ${ }^{4}$ Department of Mathematics, Aswan University, Faculty of Science, 81528, Egypt \\ ${ }^{5}$ Department of Mathematics, Salman Bin Abdul Aziz University, College of Science and Humanity Studies, AL-Kharj, \\ Saudi Arabia
}

\begin{abstract}
The effects of viscous dissipation and magnetic field on free convection heat and mass transfer along a vertical plate embedded in a nanofluid saturated non-Darcy porous medium have been studied. The model used for the nanofluid incorporates the effects of Brownian motion and thermophoresis. The nonlinear governing equations and their associated boundary conditions are initially cast into dimensionless form by non-similarity variables. The resulting system of equations is then solved numerically by an accurate implicit finite-difference method. The numerical results are compared and found to be in good agreement with previously published results for a special case of the present investigation. The effect of the physical parameters on the flow, heat transfer and nanoparticle concentration characteristics of the model is presented through graphs and the salient features are discussed.
\end{abstract}

Keywords: Free convection, Non-Darcy porous medium, Nanofluid, Magneto-hydrodynamics, Viscous dissipation.

\section{INTRODUCTION}

The study of convective flow, heat transfer in porous media has been an active field of research as it plays a crucial role in diverse applications, such as thermal insulation, extraction of crude oil and chemical catalytic reactors etc. A number of studies have been reported in the literature focusing on the problem of heat transfer in porous media. The analysis of convective transport in a porous medium with the inclusion of non-Darcian effects has also been a matter of study in recent years. Due to its important applications in many fields, a full understanding of heat transfer by non-Darcy natural convection from a heated flat surface embedded in fluid saturated porous medium is meaningful. The inertia effect is expected to be important at a higher flow rate and it can be accounted for through the addition of a velocity squared term in the momentum equation, which is known as the Forchheimer's extension. Analysis is conducted by several authors (viz., Kairi and Murthy [1]; Prasad and Hemalatha [2]; Kairi et al. [3]; Murthy and El-Amin [4]; Srinivasacharya and RamReddy [5]; Srinivasacharya et al. [6] etc.) in order to explore the importance of convective transport of an incompressible Newtonian/Non-Newtonian fluid saturated non-Darcy porous medium. A detailed review

*Address correspondence to this author at the Department of Mathematics, National Institute of Technology, Rourkela-769008, Odisha, India; Tel: 0661-2462717; Fax: 0661-2462701;

E-mails: chittetiram@gmail.com; chramreddy@nitrkl.ac.in of convective heat transfer in Darcian and non-Darcian porous medium, including an exhaustive list of references, can be found in the book by Nield and Bejan [7].

Nanofluids are a relatively new class of fluids which consist of a base fluid with nano-sized particles $(1-100 \mathrm{~nm})$ suspended within them. These particles, generally a metal or metal oxide, increase conduction and convection coefficients, allowing for more heat transfer out of the coolant (see Choi [8]). The main advantages of nanofluids are that they are more stable and have acceptable viscosity and better wetting, spreading and dispersion properties on solid surface. In particular, nanofluids are suspensions of nanoparticles in fluids that show significant enhancement of their properties at modest nanoparticle concentrations. In this new age of energy awareness, our lack of abundant sources of clean energy and the widespread dissemination of battery operated devices, such as cell-phones and laptops, have accented the necessity for a smart technological handling of energetic resources. Nanofluids have been demonstrated to be able to handle this role in some instances as a smart fluid. The research on heat transfer in nanofluids has been receiving increased attention worldwide. Many researchers have found unexpected thermal properties of nanofluids, and have proposed new mechanisms behind the enhanced thermal properties of nanofluids. A very good collection of published papers on nanofluids can be found in the book by Das et al. [9] and in the review papers by Buongiorno [10] and Kakac and Pramuanjaroenkij [11]. Later, Serrano et al. 
[12] provided excellent examples of nanometer in comparison with milimeter and micrometer.

It is interesting to note that the Brownian motion of nanoparticles at molecular and nanoscale levels are a key nanoscale mechanism governing their thermal behaviors. In nanofluid systems, due to the size of the nanoparticles, the Brownian motion takes place, which can affect the heat transfer properties. As the particle size scale approaches to the nanometer scale, the particle Brownian motion and its effect on the surrounding liquids play an important role in the heat transfer. In view of these applications, Nield and Kuznetsov $([13,14])$ analyzed the free convective boundary layer flows in a porous medium saturated by nanofluid by taking Brownian motion and thermophoresis effects into consideration. In the first article, the authors have assumed that nanoparticles are suspended in the nanofluid using either surfactant or surface charge technology and hence they have concluded that this prevents particles from agglomeration and deposition on the porous matrix. Chamkha et al. [15] carried out a boundary layer analysis for the natural convection past an isothermal sphere in a Darcy porous medium saturated with a nanofluid. Nield and Kuznetsov [16] investigated the cross-diffusion in nanofluids, with the aim of making a detailed comparison with regular cross diffusion effects and the cross-diffusion effects peculiar to nanofluids, and at the same time investigating the interaction between these effects when the base fluid of the nanofluid is itself a binary fluid such as salty water. Recently, a boundary layer analysis for the natural convection past a horizontal plate in a porous medium saturated with a nanofluid is analyzed by Gorla and Chamkha [17].

There has been a renewed interest in Magnetohydrodynamics (MHD) flow and heat transfer in porous and clear domains due to the important effect of magnetic field on the boundary layer flow control and on the performance of many systems using electrically conducting fluid such as MHD power generators, the cooling of nuclear reactors, plasma studies, geothermal energy extractions etc. Many authors have been explored the importance of MHD in a nanofluid saturated porous medium. Chamkha and Aly [18] obtained numerical solution of steady natural convection boundary-layer flow of a nanofluid consisting of a pure fluid with nanoparticles along a permeable vertical plate in the presence of magnetic field. Hamada et al. [19] reported the similarity reductions for problems of magnetic field effects on free convection flow of a nanofluid past a semi-infinite vertical flat plate. MHD convective flow of a nanofluid over a stretching sheet has been investigated by Matin et al. [20]. As has been pointed out by others, magnetic nanofluid has many applications: magnetofluidic leakage-free rotating seals, magnetogravimetric separations, acceleration/ inclinations sensors, aerodynamic sensors (differential pressure, volumic flow), nano/micro-structured magnetorheological fluids for semiactive vibration dampers, biomedical applications in plant genetics and veterinary medicine. Recently, Magneto-hydrodynamic boundary layer flow of a nanofluid over an exponentially stretching sheet has been studied by Ferdows et al. [21].

Viscous dissipation acts as a heat source and generates appreciable temperature in the medium. In the porous medium, it is interpreted as the rate at which mechanical energy is converted into heat in a viscous fluid per unit volume. The effect of viscous dissipation has received little attention in the past. Viscous dissipation is of significance in natural convection in various devices that are subjected to large variations of gravitational force or that operate at high rotational speeds (see Gebhart [22]). In the study of flow past a vertical porous plate, Takhar and Beg [23] have modeled the viscous dissipation in the porous medium and on the other hand, Murthy and Singh [24] have modeled the flow of an incompressible fluid in a saturated porous medium, with the effect of viscous dissipation included. The effect of viscous dissipation on the development of the boundary layer flow from a cold vertical surface embedded in a Darcian porous medium is investigated by Rees et al. [25]. Nield [26] made a critical review of recent studies of the modeling of viscous dissipation in a saturated porous medium, with applications to either forced convection or natural convection. Also, he has discussed alternative forms of the viscous dissipation function (also see the references cited therein).

Several researchers have been directed on the effect of viscous dissipation in porous media in regular fluids, but there is very limited literature available on the study of viscous dissipation in nanofluids about different surface geometries. Laminar two dimensional forced convective heat transfer of $\mathrm{Al}_{2} \mathrm{O}_{3}$-water nanofluid in a horizontal microchannel has been studied numerically by Ramiar and Ranjbar [27], considering axial conduction, viscous dissipation and variable properties effects. Uddin et al. [28] analyzed the influences of viscous dissipation on free convective boundary layer flow of a non-Newtonian power law nanofluid over an isothermal vertical flat plate embedded in a porous medium. The effects of suction, viscous dissipation, thermal radiation and thermal diffusion on a boundary layer flow of nanofluids over a moving flat plate has been discussed by Motsumi and Makinde [29].

But, very little attention has been paid to study the significance of combined effects of MHD and viscous dissipation on free convection in a nanofluid saturated nonDarcy porous medium. These nanofluids appear to have a very high thermal conductivity and may be able to meet the rising demand as an efficient heat transfer agent. In recent past, scientists and engineers have started showing interest in the study heat transfer characteristics of these nanofluids. Kameswaran et al. [30] investigated the convective heat and mass transfer in nanofluid flow over a stretching sheet subject to hydromagnetic, viscous dissipation, chemical reaction and Soret effects. Recently, Murthy et al. [31] investigated the influence of the prominent Soret effect on double-diffusive free convection heat and mass transfer in the boundary layer region of a semi-infinite inclined flat plate in a nanofluid saturated non-Darcy porous medium. But a clear picture about the heat transfer through these nanofluids is yet to emerge.

The effects of viscous dissipation and magnetic field on free convection along a vertical plate in a non-Darcy porous medium saturated with a nanofluid have not been reported in the literature. The purpose of the present work is to investigate the viscous dissipation and MHD effects on free convection heat and nanoparticle mass transfer along a vertical plate in a nanofluid saturated non-Darcy porous 


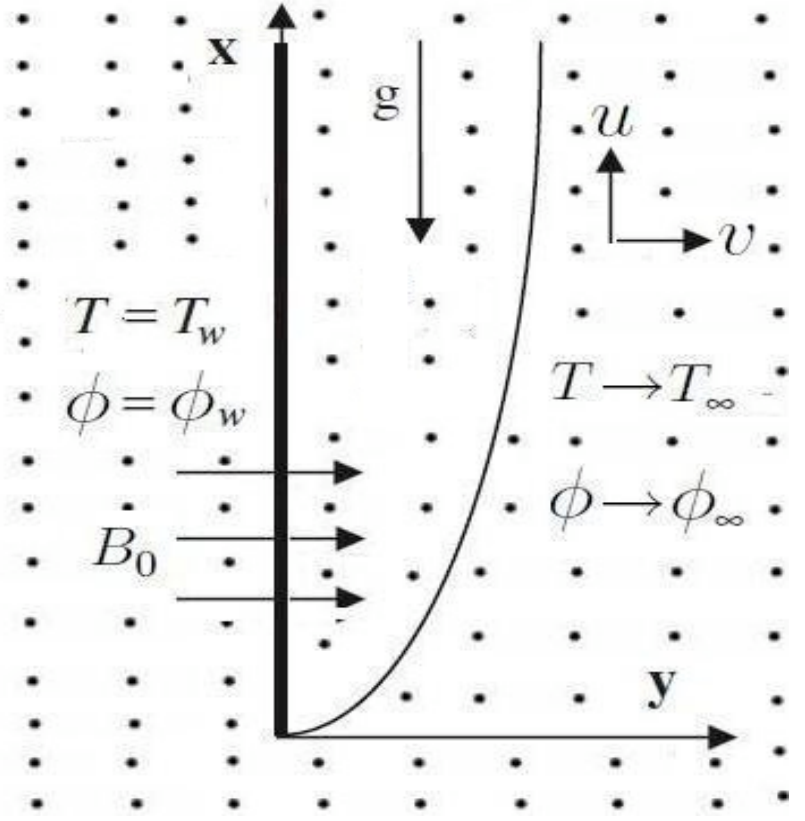

Fig. (1). Physical model and coordinate system.

medium with uniform wall temperature and nanoparticle concentration. The implicit, iterative finite-difference method discussed by Blottner [32] is employed to solve the nonlinear system of this particular problem. The effects of magnetic, viscous dissipation and non-Darcy parameters are examined and are displayed through graphs. The results are compared with relevant results in the existing literature and are found to be in good agreement.

\section{MATHEMATICAL FORMULATION}

Consider the free convection flow of an electrically conducting fluid from vertical plate embedded in a nanofluid saturated non-Darcy porous medium. A uniform magnetic field is applied normal to the plate. The magnetic Reynolds number is assumed to be small so that the induced magnetic field can be neglected. The porous medium is considered to be homogeneous and isotropic (i.e., uniform with a constant porosity and permeability) and is saturated with a fluid which is in local thermodynamic equilibrium with the solid matrix. The fluid has constant properties except the density in the buoyancy term of the balance of momentum equation. The fluid flow is moderate, so the pressure drop is proportional to the linear combination of fluid velocity and the square of velocity (Forchheimer flow model is considered). Also, the fluid flow is steady, laminar and two dimensional. We choose the coordinate system such that $\mathrm{x}$ axis is along the vertical plate and y-axis is normal to the plate. The physical model and coordinate system are shown in Fig. (1). The plate is maintained at uniform wall temperature $T_{w}$ and nanoparticle concentration $\phi_{w}$. These values are assumed to be greater than the ambient temperature $T_{\infty}$ and nanoparticle concentration $\phi_{\infty}$ at any arbitrary reference point in the medium (inside the boundary layer).

By employing the Oberbeck-Boussinesq and the standard boundary layer approximations, and making use of the
Darcy-Forchheimer model (Nield and Bejan [7]), the governing equations for the nanofluid flow problem under investigation are given by

$$
\begin{aligned}
& \frac{\partial u}{\partial x}+\frac{\partial v}{\partial y}=0 \\
& \left(1+\frac{\sigma \mu_{e}^{2} B_{0}^{2} K}{\mu}\right) u+\frac{c \sqrt{K}}{\left(\mu / \rho f_{\infty}\right)} u^{2}=\frac{K\left(1-\phi_{\infty}\right) \rho f_{\infty} g \beta}{\mu} \\
& \left(T-T_{\infty}\right)-\frac{\left(\rho_{P}-\rho f_{\infty}\right) g K}{\mu}\left(\phi-\phi_{\infty}\right) \\
& u \frac{\partial T}{\partial x}+v \frac{\partial T}{\partial y}=\alpha_{m} \frac{\partial^{2} T}{\partial y^{2}}+J\left[D_{B} \frac{\partial \phi}{\partial y} \frac{\partial T}{\partial y}+\frac{D_{T}}{T_{\infty}}\left(\frac{\partial T}{\partial y}\right)^{2}\right] \\
& +\frac{v}{k_{m} C_{p}} u\left[u+\frac{c \sqrt{K}}{\left(\mu / \rho f_{\infty}\right.} u^{2}\right]
\end{aligned}
$$

$\frac{1}{\phi}\left(u \frac{\partial \phi}{\partial x}+v \frac{\partial \phi}{\partial y}\right)=D_{B} \frac{\partial^{2} \phi}{\partial y^{2}}+\frac{D_{T}}{T_{\infty}} \frac{\partial^{2} T}{\partial y^{2}}$

where $u$ and $v$ are the Darcy velocity components in the $x$ and $y$ directions, respectively, $T$ is the temperature, $\phi$ is the nanoparticle concentration, $g$ is the acceleration due to gravity, $K$ is the permeability, $c$ is the empirical constant associated with the Forchheimer porous inertia term, $\sigma$ is the electrical conductivity of the fluid, $\mu_{e}$ is the magnetic permeability, $B_{0}$ is the strength of the magnetic field, $\varphi$ is the porosity, $\alpha_{m}=k_{m} /(\rho c)_{f}$ is the thermal diffusivity of the fluid, $v=\mu / \rho f_{\infty}$ is the kinematic viscosity coefficient and $J=\varphi(\rho c)_{p} /(\rho c)_{f}$. Further, is the density of the base fluid and $\rho, \mu, k_{m}$ and $\beta$ are the density, viscosity, thermal conductivity and volumetric thermal expansion coefficient of the nanofluid, while $\rho_{p}$ is the density of the nanoparticles, $(\rho c)_{f}$ is the heat capacity of the fluid and $(\rho c)_{p}$ is the effective heat capacity of the nanoparticle material. The coefficients that appear in Eqs. (3) - (4) are the Brownian diffusion coefficient $D_{B}$, the thermophoretic diffusion coefficient $D_{T}$ and the last term in Eq. (3) is the viscous dissipation term, which can be approximated as the work done by the surface and the body forces acting on the pore (see Murthy and Singh [24]). For detailed derivation of the Eqs. (1) - (4), one can refer the papers by Buongiorno [10] and Nield and Kuznetsov ([13, 14]).

The associated boundary conditions are

$$
\begin{aligned}
& v=0, T=T_{w}, \phi=\phi_{w} \text { at } y=0 \\
& u=0, T=T_{\infty}, \phi=\phi_{\infty} \text { as } y \rightarrow \infty
\end{aligned}
$$


where the subscripts $w$ and $\infty$ indicate the conditions at the wall, and at the outer edge of the boundary layer respectively.

Introducing the following non-dimensional transformations

$\eta=\frac{y}{x} R a_{x}^{1 / 2}, \psi(x, \eta)=\alpha_{m} R a_{x}^{1 / 2} f(x, \eta), \theta(x, \eta)=\frac{T-T_{\infty}}{T_{w}-T_{\infty}}$,

$S(x, \eta)=\frac{\phi-\phi_{\infty}}{\phi_{w}-\phi_{\infty}}$

where $R a_{x}=\frac{\left(1-\phi_{\infty}\right) \rho f_{\infty} g K \beta\left(T_{w}-T_{\infty}\right) x}{\mu \alpha_{m}}$ is the local Rayleigh number.

In view of the continuity Eq. (1), we introduce the stream function $\psi$ by

$$
u=\frac{\partial \psi}{\partial y}, v=-\frac{\partial \psi}{\partial x}
$$

Substituting Eq. (7) in Eqs. (2)-(4) and then using the non-dimensional transformations (6), we get the following system of non-dimensional equations:

$(1+H a) f^{\prime}+G r f^{\prime 2}=\theta-\mathrm{N} r . S$

$\theta^{\prime \prime}+\frac{1}{2} f \theta^{\prime}+N b \cdot \theta^{\prime} S^{\prime}+N t \cdot \theta^{\prime 2}+\varepsilon f^{\prime 2}\left(1+G r f^{\prime}\right)=\varepsilon\left(f^{\prime} \frac{\partial \theta}{\partial \varepsilon}-\theta^{\prime} \frac{\partial f}{\partial \varepsilon}\right)$

$S^{\prime \prime}+\frac{1}{2} L e . f S^{\prime}+\frac{N t}{N b} \theta^{\prime \prime}=\operatorname{Le} \varepsilon\left(f^{\prime} \frac{\partial S}{\partial \varepsilon}-S^{\prime} \frac{\partial f}{\partial \varepsilon}\right)$

where the primes indicate partial differentiation with respect to $\eta$ alone, $G r=\frac{c \sqrt{K}}{v} \frac{\alpha_{m}}{x} R a_{x}$ is the non-Darcy parameter, $L e=\frac{\alpha_{m}}{\varphi D_{B}}$ is the Lewis number, $N r=\frac{\left(\rho_{p}-\rho f_{\infty}\right)\left(\phi_{w}-\phi_{\infty}\right)}{\rho f_{\infty} \beta\left(1-\phi_{\infty}\right)\left(T_{w}-T_{\infty}\right)}$ is the buoyancy parameter, $N b=\frac{J D_{B}\left(\phi_{w}-\phi_{\infty}\right)}{\alpha_{m}}$ is the Brownian motion parameter, $N t=\frac{J D_{T}}{\alpha_{m} T_{\infty}}\left(T_{w}-T_{\infty}\right)$ is the thermophoresis parameter, $H a=\frac{\sigma \mu_{e}^{2} B_{0}^{2} K}{\mu}$ is the magnetic parameter and $\varepsilon=\frac{\left(1-\phi_{\infty}\right) g \beta x}{C_{p}}$ is the viscous dissipation parameter (i.e.,

Eckert number). For most situations the Darcy number is small, so viscous dissipation is important at even modest values of the Eckert number. The circumstances in which viscous dissipation is important are those involving flows of relatively large velocity. The author believes that the results in this paper are likely to be applicable in the context of particle bed nuclear reactors.

The boundary conditions (5) in terms of $f, \theta$ and $S$ become

$$
\begin{aligned}
& \eta=0: f(\varepsilon, 0)=-2 \varepsilon \frac{\partial f}{\partial \varepsilon}, \theta(\varepsilon, 0)=1, S(\varepsilon, 0)=1 \\
& \eta \rightarrow \infty: f^{\prime}(\varepsilon, \infty) \rightarrow 0, \theta(\varepsilon, \infty) \rightarrow 0, S(\varepsilon, \infty) \rightarrow 0
\end{aligned}
$$

If $\varepsilon=0, H a=0$ and $G r=0$, the problem reduces to free convective boundary-layer flow in a porous medium saturated by a nanofluid. In the limit $\varepsilon \rightarrow 0$, the governing equations (8)-(10) reduce to the corresponding equations for a non-Darcy porous medium saturated with nanofluids in the presence of MHD effects. Furthermore, if $N b \rightarrow 0, N t=N r=0, \varepsilon=0, H a=0$ and $S(\eta) \rightarrow 0$ (i.e., for the regular Newtonian fluid), Eqs. (8) - (10) governing the nonDarcy porous medium saturated with a nanofluid reduce to the non-Darcy free convection from vertical isothermal surfaces in saturated porous media in the absence of viscous dissipation and MHD effects.

\section{HEAT AND MASS TRANSFER COEFFICIENTS}

The primary objective of this study is to estimate the parameters of engineering interest in fluid flow, heat and nanoparticle mass transport problems are the Nusselt number $N u_{x}$, nanoparticle Sherwood number $S h_{x}$. These parameters characterize the wall heat and nanoparticle mass transfer rates, respectively.

The local heat and local nanoparticle mass fluxes from the vertical plate can be obtained from

$q_{w}=-k_{m}\left(\frac{\partial T}{\partial y}\right)_{y=0}, \quad q_{m}=-D_{B}\left(\frac{\partial \phi}{\partial y}\right)_{y=0}$

The dimensionless local Nusselt number $N u_{x}=\frac{q_{w} x}{k_{m}\left(T_{w}-T_{\infty}\right)}$ and local nanoparticle Sherwood number $S h_{x}=\frac{q_{m} x}{D_{B}\left(\phi_{w}-\phi_{\infty}\right)}$ are given by

$\frac{N u_{x}}{R a_{x}^{1 / 2}}=-\theta^{\prime}(\varepsilon, 0)$ and $\frac{S h_{x}}{R a_{x}^{1 / 2}}=-S^{\prime}(\varepsilon, 0)$

The effects of the various parameters involved in the investigation on these coefficients are discussed in the Results and Discussion section.

\section{NUMERICAL METHOD}

The reduced flow governing Equations (8-10) are nonlinear, coupled, ordinary differential equations, which possess no closed-form solution. Therefore, they must be solved numerically subject to the boundary conditions given by Eq. (11). The implicit, iterative finite-difference method discussed by Blottner [32] has proven to be adequate for the solution of this type of equations. For this reason, this method is employed in the present work. Eqs. (8)-(10) are discretized using three-point central difference quotients. This converts the differential equations into linear sets of algebraic equations, which can be readily solved by the well known Thomas algorithm (see Blottner [32]). On the other hand, Eqs. (8)-(10) are discretized and solved subject to the 
Table 1. Comparison of Dimensionless Similarity Functions $\theta^{\prime}(\eta)$ and $f^{\prime \prime}(\eta)$ for Free Convection Along a Vertical Flat Plate in non-Darcy Porous Medium with $N b \rightarrow 0, N t=N r=0, \varepsilon=0, H a=0$ and $S(\eta) \rightarrow 0$ (Plumb and Huenefeld [33])

\begin{tabular}{|c|c|c|c|c|}
\hline & $\boldsymbol{\theta}^{\prime}(\mathbf{0})$ & & $\boldsymbol{f}^{\prime \prime}(\mathbf{0})$ \\
\hline $\boldsymbol{G} \boldsymbol{r}$ & Plumb and Huenefeld [33] & Present & Plumb and Huenefeld [33] & Present \\
\hline \hline 0.00 & 0.44390 & 0.44374 & 1.00000 & 0.99020 \\
\hline 0.01 & 0.44232 & 0.44216 & 0.91608 & 0.99019 \\
\hline 0.10 & 0.42969 & 0.42950 & 0.61803 & 0.91608 \\
\hline 1.00 & 0.36617 & 0.36575 & 0.27016 & 0.61803 \\
\hline 10.00 & 0.25126 & 0.25065 & 0.09512 & 0.09512 \\
\hline 100.00 & 0.15186 & 0.15145 & & 0.000 \\
\hline
\end{tabular}

appropriate boundary conditions by the trapezoidal rule. The computational domain in the $\eta$-direction was made up of 196 non-uniform grid points. It is expected that most changes in the dependent variables occur in the region close to the plate where viscous effects dominate. However, small changes in the dependent variables are expected far away from the plate surface. For these reasons, variable step sizes in the $\eta$-direction are employed. The initial step size $\Delta \eta_{1}$ and the growth factor $K^{*}$ employed such that $\Delta \eta_{i+1}=K^{*} \Delta \eta_{i}$ (where the subscript $i$ indicates the grid location) were $10^{-3}$ and 1.0375 , respectively. These values were found (by performing many numerical experimentations) to give accurate and grid-independent solutions. The solution convergence criterion employed in the present work was based on the difference between the values of the dependent variables at the current and the previous iterations. When this difference reached $10^{-5}$, the solution was assumed converged and the iteration process was terminated.

With $\quad N b \rightarrow 0, N t=N r=0, \quad \varepsilon=0, H a=0 \quad$ and $\quad S(\eta) \rightarrow 0$ (i.e., for the regular Newtonian fluid), Eqs. (8) - (10) governing the present investigation of nanofluid saturated non-Darcy porous medium (with isothermal boundary) reduces to those limiting case of free convection flow Plumb and Huenefeld [33] who investigated non-Darcy natural convection from vertical isothermal surfaces in saturated porous media in the absence of viscous dissipation and MHD effects. Also, the results have been compared with Plumb and Huenefeld [33] and it is found that they are in good agreement as shown in Table 1. Therefore, the developed code can be used with great confidence to study the problem considered in this paper.

\section{RESULTS AND DISCUSSION}

We have computed the solutions for the dimensionless velocity, temperature and nanoparticle volume fraction functions and heat and nanoparticle mass transfer rates as shown graphically in Figs. (2-10). The effects of viscous dissipation parameter $\mathcal{E}$, magnetic parameter $H a$, non-Darcy parameter $G r$, Brownian motion parameter $\mathrm{Nb}$, thermophoresis parameter $N t$, Lewis number $L e$ and buoyancy ration $N r$ have been discussed.

The dimensionless velocity distribution for different values of non-Darcy parameter $\mathrm{Gr}$ and Brownian motion $\mathrm{Nb}$ with $H a=2.0, N r=0.1 N t=0.1, \varepsilon=0.5 \quad$ and $L e=10 \quad$ is depicted in Fig. (2a). Since $G r$ represent the inertial drag, thus an increase in the non-Darcy parameter increases the resistance to the flow and so a decrease in the fluid velocity ensues. Here $G r=0$ represents the case where the flow is Darcian. The velocity is maximum in this case due to the total absence of inertial drag. With $N b=0$, there is no thermal transport due to buoyancy effects created as a result of nanoparticle concentration gradients. It is observed that the momentum boundary layer thickness increases with the increase of $N b$. The dimensionless temperature for different values of non-Darcy parameter $\mathrm{Gr}$ and Brownian motion $\mathrm{Nb}$ for the fixed values of other parameters is displayed in Fig. (2b). An increase in non-Darcy parameter $G r$, increase temperature values, since as the fluid is decelerated, energy is dissipated as heat and serves to increase temperatures. As such the temperature is minimized for the lowest value of $G r$ and maximized for the highest value of $G r$ as shown in Fig. (2b). As the parameter $N b$ increase, the temperature increases for the specified conditions. As expected, the boundary layer profile for the temperature function is essentially the same form as in the case of a regular (Newtonian) fluid. Fig. (2c) depicts that the dimensionless volume fraction for different values of non-Darcy parameter $\mathrm{Gr}$ and Brownian motion $\mathrm{Nb}$ for fixed values of other parameters. As the parameter $G r$ increases, the volume fraction profile increases but the nanoparticle volume fraction decreases with increase in $\mathrm{Nb}$. It is noticed that the nanoparticle volume fraction increases with increase in $\mathrm{Nb}$ in the case of forced convection flow. The increase in nonDarcy parameter reduces the intensity of the flow but enhance the thermal and nanoparticle volume fraction boundary layer thicknesses.

In Fig. (3), the non-dimensional heat transfer coefficient plotted against the viscous dissipation parameter $\varepsilon$ for different values of non-Darcy parameter $G r$ and Brownian motion $N b$ with $H a=2.0, N r=0.1 N t=0.1$ and $L e=10$. It indicates that heat transfer rate decreases with the viscous dissipation parameter. To increase the fluid motion we have considered viscous dissipation term. From this term we obtained dimensionless parameter $\mathcal{E}$. This parameter is called the fluid motion controlling parameter. It may be noted that $\varepsilon=0$ corresponds to the case of absence of viscous dissipation. Furthermore, the presence of viscous dissipation in the energy equation acts as an internal heat source due to the action of viscous stresses. Therefore, the 

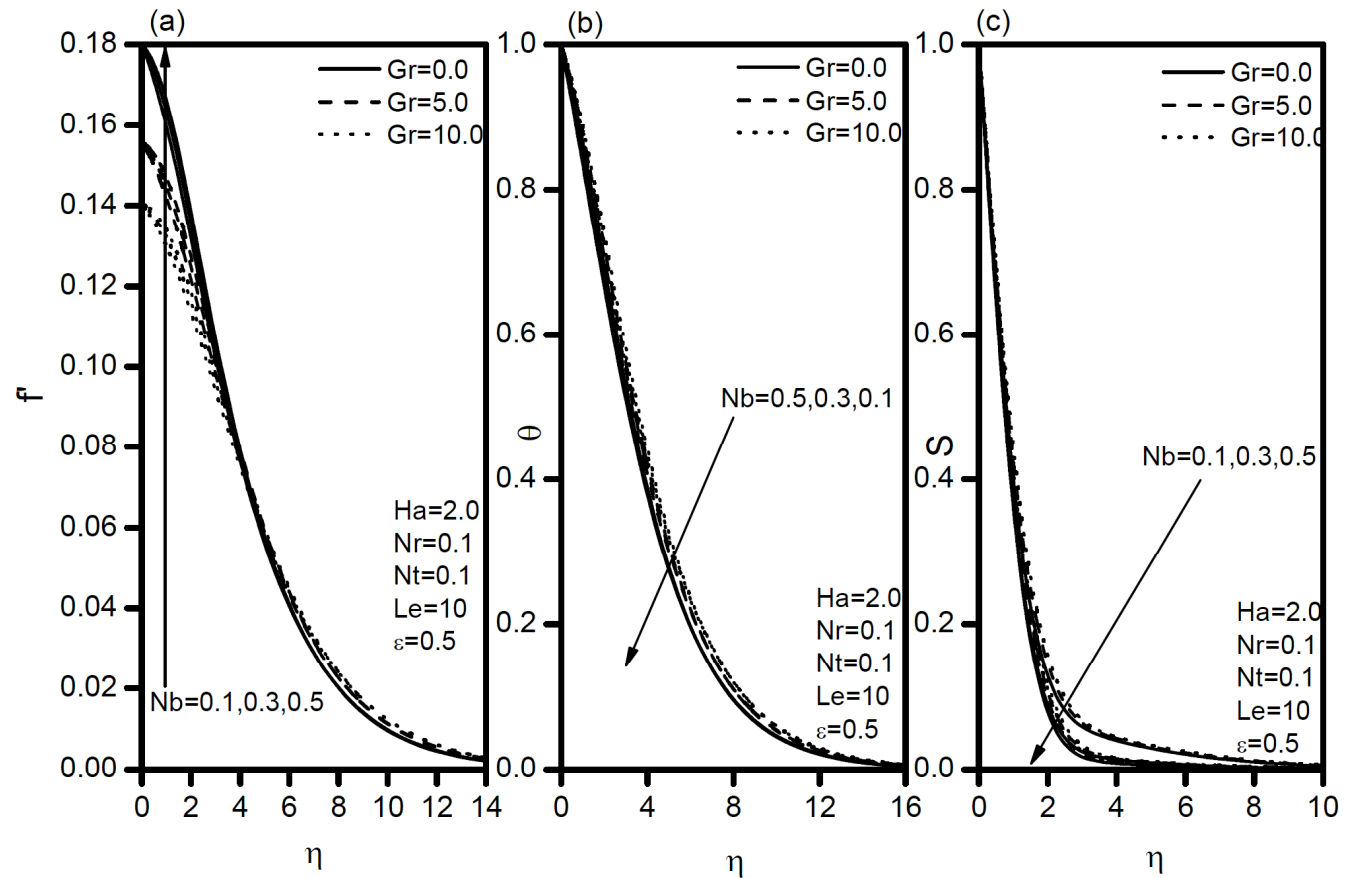

Fig. (2). Effects of non-Darcy parameter Gr and Brownian motion $\mathrm{Nb}$ on (a) Velocity, (b) Temperature, and (c) Volume fraction profiles.

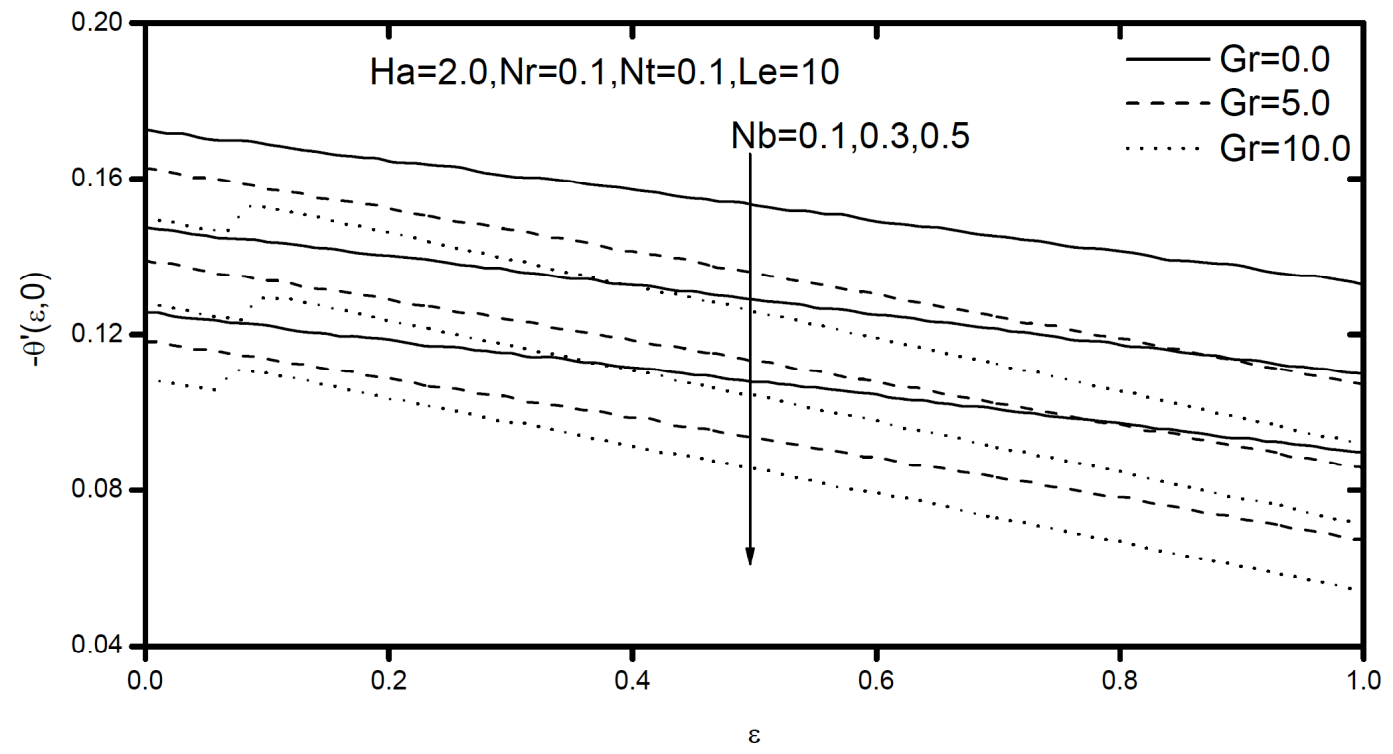

Fig. (3). Variation of non-dimensional heat transfer coefficient with $\varepsilon$ for different values of $\mathrm{Gr}$ and $\mathrm{Nb}$.

heat transfer rate is at a lower level when this effect is considered $(\varepsilon \neq 0)$ than that when this effect is neglected $(\varepsilon=0)$. Also, the results indicated that increases in $\mathrm{Gr}$ decreases the heat transfer coefficient. Similar trend can be observed with the increasing values of $\mathrm{Nb}$. The nondimensional nanoparticle mass transfer coefficient is plotted against the viscous dissipation parameter $\varepsilon$ for different non-Darcy parameter $\mathrm{Gr}$ and Brownian motion $\mathrm{Nb}$ with $H a=2.0, N r=0.1 N t=0.1$ and $L e=10$ in Fig. (4). It is evident from this figure that for increasing values of $G r$ the non-dimensional nanoparticle mass transfer coefficient decreases whereas with increasing values of $\mathrm{Nb}$ nondimensional nanoparticle mass transfer coefficient increases. Finally, the non-dimensional mass transfer coefficient increases with increasing values of $\mathcal{E}$. Hence the non-Darcy parameter has an important role in controlling the flow field.
The variations of the non-dimensional velocity, temperature and nanoparticle concentration with magnetic parameter $\mathrm{Ha}$ and Lewis number $\mathrm{Le}$ for fixed values of other parameters are shown in Fig. (5). It can be observed from Fig. (5a) that the velocity of the fluid is decreased with increase in the value of the magnetic parameter. This is due to the fact that the introduction of a transverse magnetic field, normal to the flow direction, has a tendency to create the drag known as the Lorentz force which tends to resist the flow. Hence, the horizontal velocity profiles decrease as the magnetic parameter $\mathrm{Ha}$ increases. It can be found from Fig. (5b) that increases in the value of the magnetic parameter increases the temperature of the fluid in the medium. It can be seen from Fig. (5c) that the nanoparticle volume fraction of the fluid is increased by increasing the value of the magnetic parameter. As explained above, the transverse 


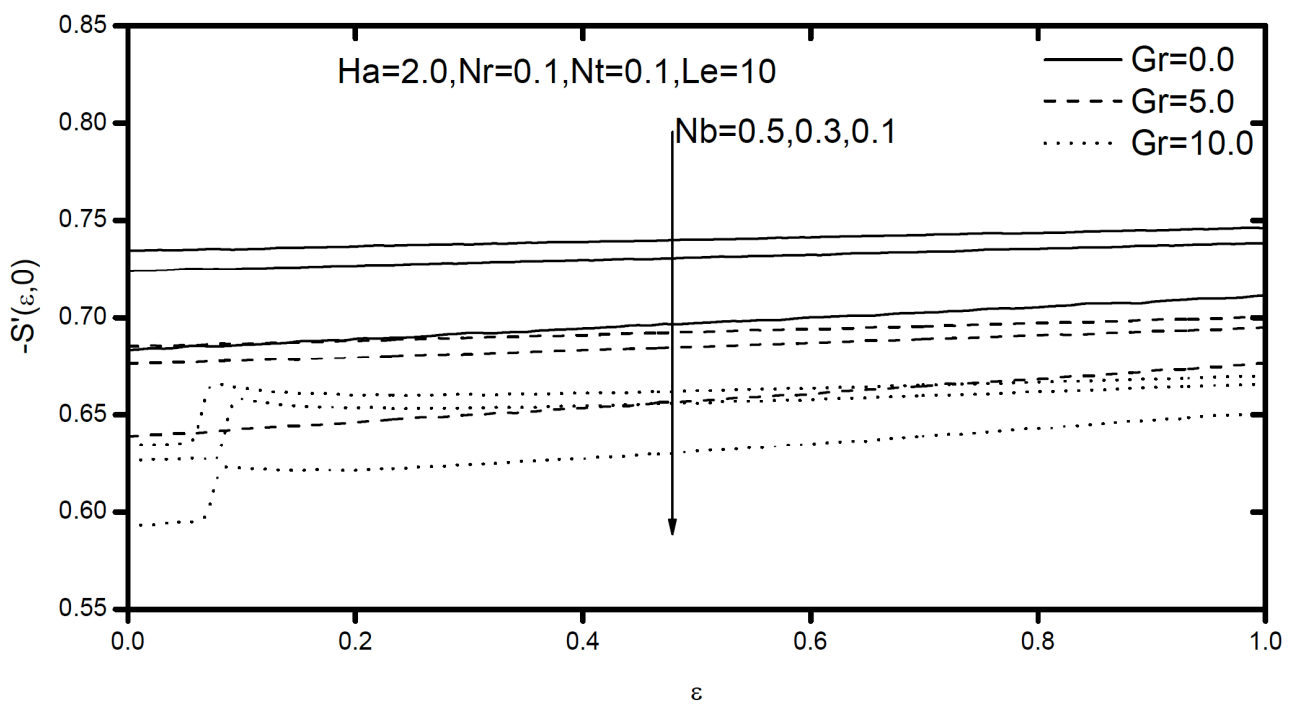

Fig. (4). Variation of non-dimensional nanoparticle mass transfer coefficient with $\varepsilon$ for different values of $\mathrm{Gr}$ and $\mathrm{Nb}$.
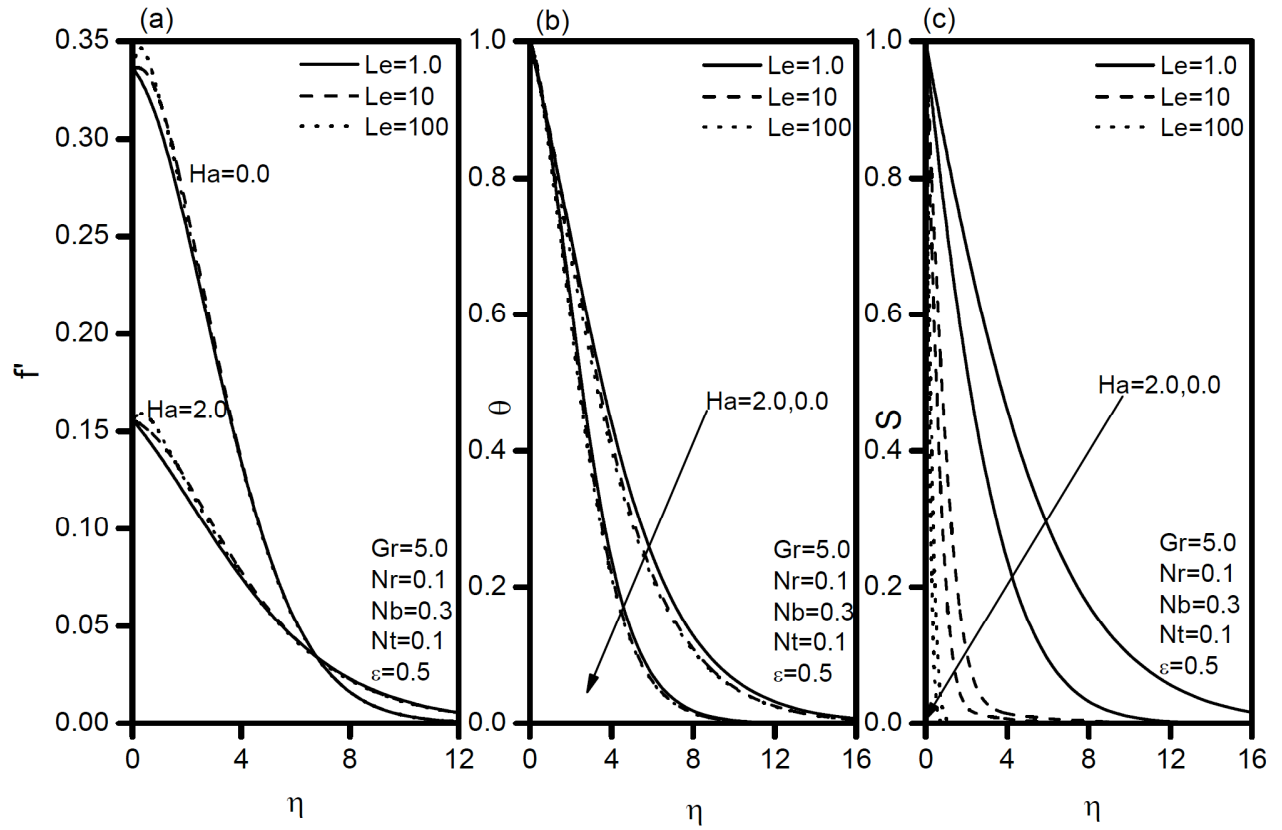

Fig. (5). Effects of Lewis number and magnetic parameter on (a) Velocity, (b) Temperature, and (c) Volume fraction profiles.

magnetic field gives rise to a resistive force known as the Lorentz force of an electrically conducting fluid. This force makes the fluid experience a resistance by increasing the friction between its layers and thus increases its temperature and nanoparticle volume fraction. It is noticed from Fig. (5) that an increase in the Lewis number $L e$ results in an increase in the velocity but decrease in the temperature and volume fraction within the boundary layer. The present analysis shows that the flow field is appreciably influenced by the Lewis number $L e$.

The effects of a magnetic field and Lewis number on the wall heat and nanoparticle mass transfer rates are shown in Fig. (6 and 7). The influence of a magnetic field is to reduce both the wall heat and nanoparticle mass transfer rates. The similar behavior can be seen in the case of Lewis number also. Further, the non-dimensional heat transfer rate decreases but nanoparticle mass transfer rate increases with increasing values of $\varepsilon$. The combined effect of the magnetic field and the Lewis number (see Fig. 5) is to generate more heat in the boundary layer region and hence to reduce the wall heat transfer rate.

Fig. (8) is prepared to present the effect of the nanoparticle buoyancy ratio $\mathrm{Nr}$ and thermophoresis $\mathrm{Nt}$ on the velocity, temperature and volume fraction distributions. It is observed that the momentum boundary layer thickness increases with the increase of $N t$ but decreases with the increase of $N r$. As the parameters $N t$ and $N r$ increase, the temperature increases for the specified conditions. As expected, the boundary layer profile for the temperature function is essentially the same form as in the case of a regular (Newtonian) fluid. The nanoparticle volume fraction increases with increase in both $N t$ and $N r$. We notice that, positive $N t$ indicates a cold surface while negative to a hot surface. For hot surfaces, thermophoresis tends to blow the nanoparticle volume fraction boundary layer away from the surface since a hot surface repels the sub-micron sized 


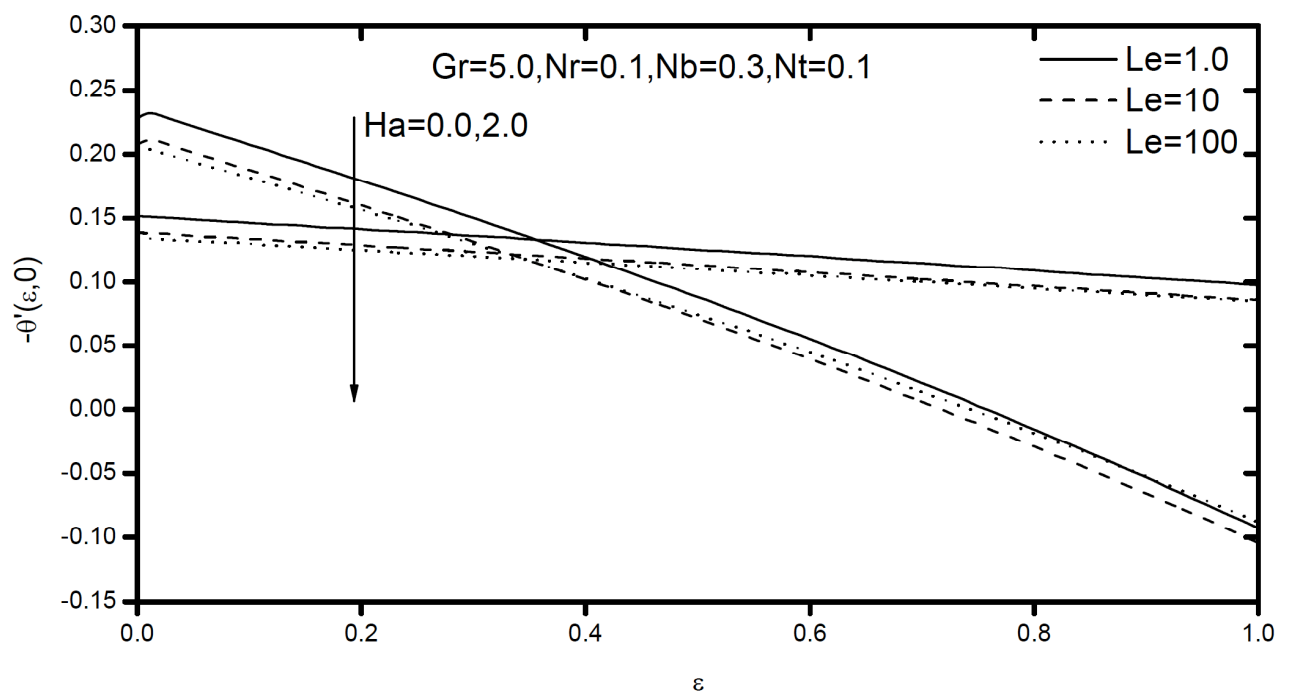

Fig. (6). Variation of non-dimensional heat transfer coefficient versus $\varepsilon$ for different values of Ha and Le.

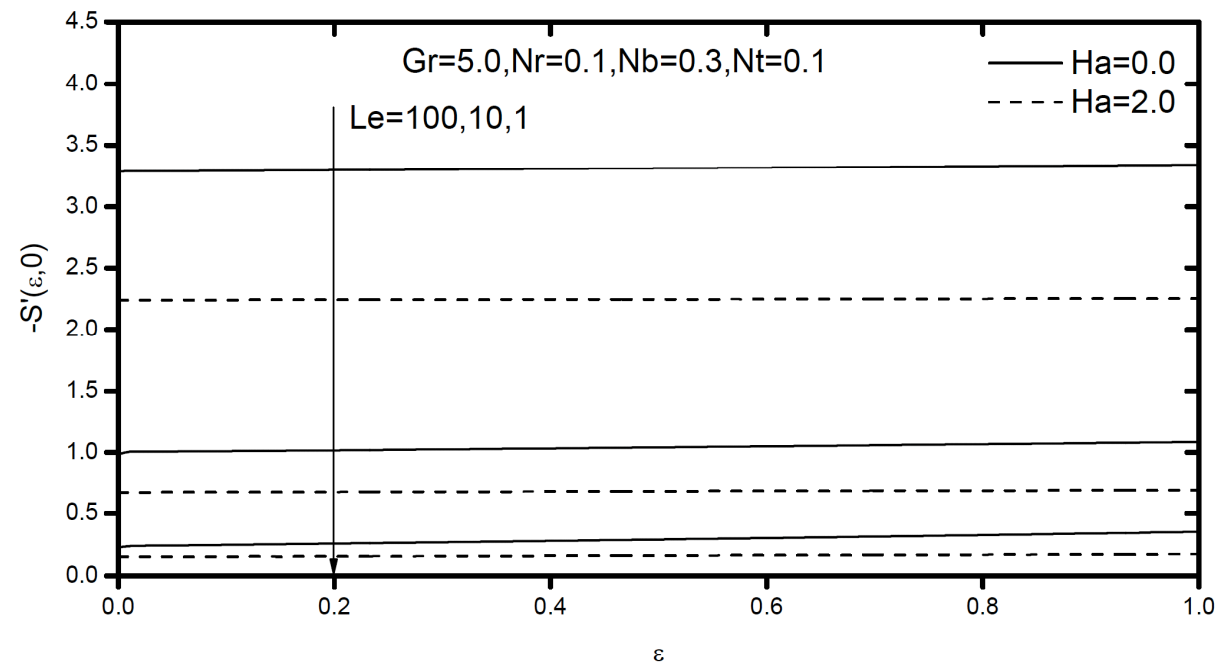

Fig. (7). Variation of non-dimensional nanoparticle mass coefficient versus $\varepsilon$ for different values of Ha and Le.
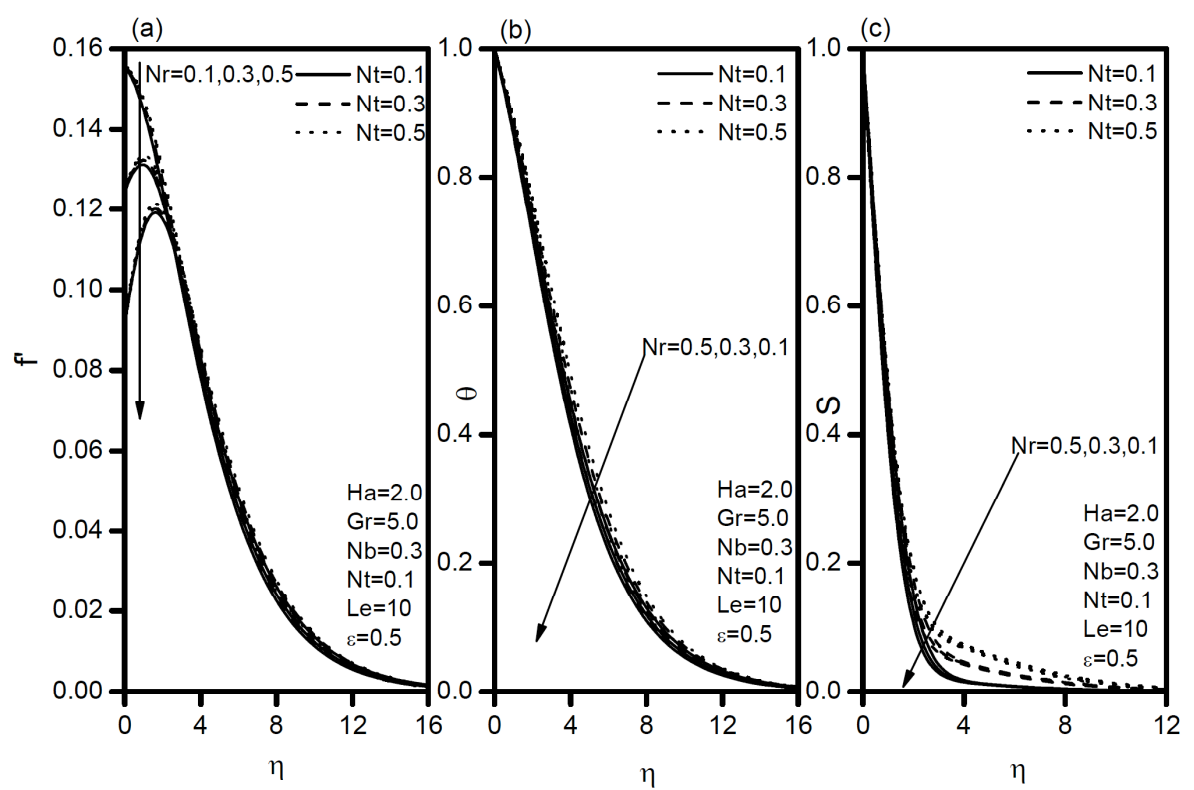

Fig. (8). Effects of nanoparticle buoyancy ratio and thermophoresis parameters on (a) Velocity, (b) Temperature, and (c) Volume fraction profiles. 


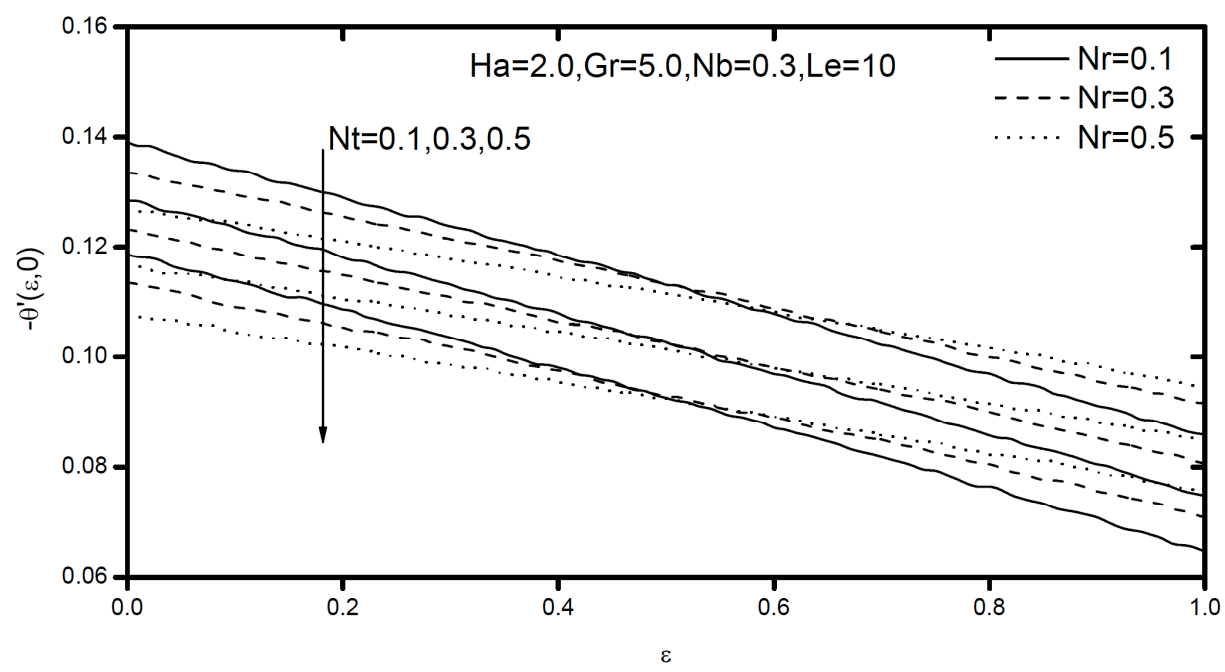

Fig. (9). Variation of non-dimensional heat transfer coefficient versus $\varepsilon$ for different values of $\mathrm{Nt}$ and $\mathrm{Nr}$.

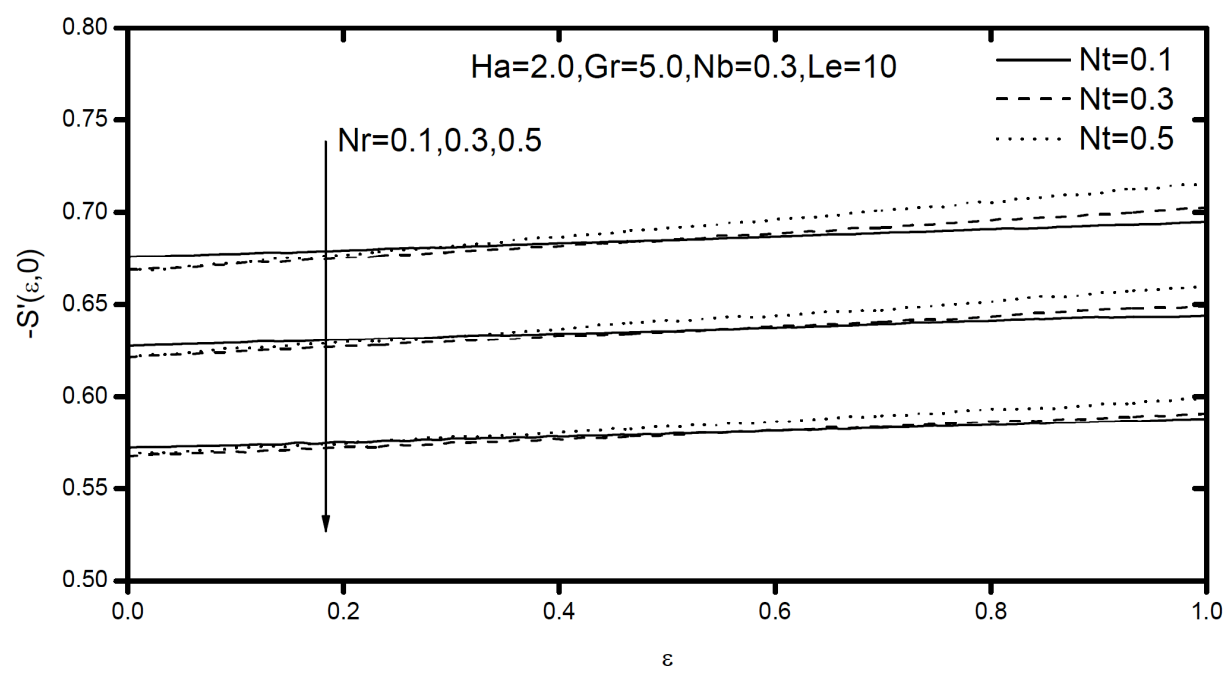

Fig. (10). Variation of non-dimensional nanoparticle mass transfer coefficient versus $\varepsilon$ for different values of $\mathrm{Nt}$ and $\mathrm{Nr}$.

particles from it, thereby forming a relatively particle-free layer near the surface.

The non-dimensional heat transfer coefficient decreases with increasing values of thermophoresis parameters but location of the maximum heat transfer rate moves farther away from the wall with increasing values of nanoparticle buoyancy ratio as shown in Fig. (9). As $\varepsilon$ increases, it can be observed from Fig. (9) that the maximum of nondimensional heat transfer rate decreases in amplitude. Further, it can be seen from Fig. (10) that the increasing values of non-dimensional mass transfer coefficient with an increase in both viscous dissipation parameter and nanoparticle buoyancy ratio but location of the maximum mass transfer rate moves farther away from the wall with thermophoresis parameter. Finally, the non-dimensional heat transfer rate decreases but nanoparticle mass transfer rate increases with increasing values of $\varepsilon$.

\section{CONCLUSIONS}

In this paper, a boundary layer analysis for free convection heat and mass transfer along a vertical plate in a nanofluid saturated non-Darcy porous medium in the presence of magnetic field and viscous dissipation effects is presented. The wall is subjected to uniform temperature and concentration conditions. Using the dimensionless variables, the governing equations are transformed into a set of nonlinear parabolic equations where numerical solution has been presented using the implicit, iterative finite difference method discussed by Blottner [32] for a wide range of parameters. Higher values of the non-Darcy parameter $\mathrm{Gr}$ results in lower velocity distribution, heat and nanoparticle mass transfer rates but higher temperature and nanoparticle volume fraction distributions. An increase in viscous dissipation parameter $\varepsilon$, raise in velocity and temperature distribution, nanoparticle mass transfer rate but fall in nanoparticle volume fraction distribution and heat transfer rate. The higher values of the magnetic parameter $\mathrm{Ha}$ resulting in lower velocity distribution and heat and nanoparticle mass transfer rate but higher temperature and nanoparticle volume fraction distributions in the boundary layer. An increase in Brownian motion parameter $\mathrm{Nb}$, enhanced velocity and temperature distributions and nanoparticle mass transfer rate but reduced nanoparticle volume friction and heat transfer rate in boundary layer. An increase in thermophoresis parameter $N t$, increase in velocity, temperature, nanoparticle volume fraction distributions but decrease in non-dimensional heat and 
nanoparticle mass transfer rates in boundary layer. The results also indicate that the presence of MHD, viscous dissipation in nanofluid saturated non-Darcy porous medium influence the flow, heat and nanoparticle volume fraction of the fluid flow.

\section{CONFLICT OF INTEREST}

The authors confirm that this article content has no conflicts of interest.

\section{ACKNOWLEDGEMENTS}

Declared none.

\section{REFERENCES}

[1] R. R. Kairi, and P. V. S. N. Murthy, "Effect of melting and thermodiffusion on natural convection heat mass transfer in a nonNewtonian fluid saturated non-Darcy porous medium", The Open Transport Phenomena Journal, vol. 1, pp. 7-14, 2009.

[2] B. D. C. N. Prasad, and K. Hemalatha, "Non-Darcy mixed convection with thermal dispersion-radiation in a saturated porous medium", The Open Transport Phenomena Journal, vol. 2, pp. 109-115, 2010.

[3] R. R. Kairi, P. V. S. N. Murthy, and C. O. Ng, "Effect of viscous dissipation on natural convection in a non-Darcy porous medium saturated with non-Newtonian fluid of variable viscosity", The Open Transport Phenomena Journal, vol. 3, pp. 1-8, 2011.

[4] P. V. S. N. Murthy, and M. F. El-Amin, "Thermo-diffusion effect on free convection heat and mass transfer in a thermally linearly stratified non-Darcy porous media", The Open Transport Phenomena Journal, vol. 3, pp. 49-55, 2011.

[5] D. Srinivasacharva, and Ch. RamReddy, "Effect of double stratification on mixed convection in a micropolar fluid saturated non-Darcy porous medium", International Review of Chemical Engineering, vol. 3, pp. 222-232, 2011.

[6] D. Srinivasacharya, J. Pranitha, and Ch. RamReddy, "Magnetic and double dispersion effects on free convection in a non-Darcy porous medium saturated with power-law fluid", International Journal for Computational Methods in Engineering Science and Mechanics, vol. 13, pp. 210-218, 2012.

[7] D. A. Nield, and A. Bejan, Convection in Porous Media, $3^{\text {rd }}$ ed. Springer-Verlag, New-York, 2013.

[8] S. U. S. Choi, "Enhancing thermal conductivity of fluid with nanoparticles", D.A. Siginer, and H.P.Wang (Eds.), Developments and Applications of Non-Newtonian Flows, FED-V. 231/MD-V. 66, ASME, New York, pp. 99-105, 1995.

[9] S. K. Das, S. U. S. Choi, W. Yu, and T. Pradeep, Nanofluids: Science and Technology. Wiley, New Jersey, 2007.

[10] J. Buongiorno, "Convective transport in nanofluids", ASME Journal of Heat Transfer, vol. 128, pp. 240-250, 2006.

[11] S. Kakac, and A. Pramuanjaroenkij, "Review of convective heat transfer enhancement with nanofluids", International Journal of Heat Mass Transfer, vol. 52, pp. 3187-3196, 2009.

[12] E. Serrano, G. Rus, and J. G. Martínez, "Nanotechnology for sustainable energy", Renewable and Sustainable Energy Reviews, vol. 13 , pp. 2373-238, 2009

[13] D. A. Nield, and A. V. Kuznetsov, "The Cheng-Minkowycz problem for natural convective boundary-layer flow in a porous medium saturated by a nanofluid", International Journal of Heat and Mass Transfer, vol. 52, pp. 5792-5795, 2009a.

[14] D. A. Nield, and A. V. Kuznetsov, "Thermal instability in a porous medium layer saturated by a nanofluid", International Journal of Heat and Mass Transfer, vol. 52, pp. 5796-5801, 2009b.

[15] A. J. Chamkha, R. S. R. Gorla, and K. Ghodeswar, "Non-similar solution for natural convective boundary layer flow over a sphere embedded in a porous medium saturated with a nanofluid", Transport in Porous Media, vol. 86, pp. 13-22, 2011.

[16] D. A. Nield, and A. V. Kuznetsov, "The Cheng-Minkowycz problem for the double-diffusive natural convective boundary layer flow in a porous medium saturated by a nanofluid", International Journal of Heat and Mass Transfer, vol. 54, pp. 374-378, 2011.

[17] R. S. R. Gorla, and A. J. Chamkha, "Natural convective boundary layer flow over a horizontal plate embedded in a porous medium saturated with a nanofluid", Journal of Modern Physics, vol. 2, pp. 62-71, 2011.

[18] A. J. Chamkha, and A. M. Aly, "MHD free convection flow of a nanofluid past a vertical plate in the presence of heat generation or absorption effects", Chemical Engineering Communications, vol. 198, pp. 425-441, 2010

[19] M. A. A. Hamada, I. Pop, and A. I. Md Ismail, "Magnetic field effects on free convection flow of a nanofluid past a vertical semiinfinite flat plate", Nonlinear Analysis: Real World Applications, vol. 12, pp. 1338-1346, 2011

[20] M. H. Matin, M. R. H. Nobari, and P. Jahangiri, "Entropy analysis in mixed convection MHD flow of nanofluid over a non-linear stretching sheet", Journal of Thermal Science and Technology, vol. 7, pp. 104-119, 2012.

[21] M. Ferdows, Md. Shakhaoath Khan, Md. Mahmud Alam, and Shuyu Sun, "MHD mixed convective boundary layer flow of a nanofluid through a porous medium due to an exponentially stretching sheet", Mathematical Problems in Engineering, Article ID 408528, pp. 1-21, 2012.

[22] B. Gebhart, "Effects of viscous dissipation in natural convection", Journal of Fluid Mechanics, vol. 14, pp. 225-232, 1962.

[23] H. G. Takhar, and O. A. Beg, "Effects of transverse magnetic field, Prandtl number and Reynolds number on non-Darcy mixed convective flow of an incompressible viscous fluid past a porous vertical flat plate in a saturated porous medium", International Journal of Energy Research., vol. 21, pp. 87-100, 1997.

[24] P. V. S. N. Murthy, and P. Singh, "Effect of viscous dissipation on a non-Darcy natural convection regime", International Journal of Heat and Mass Transfer, vol. 40, pp. 1251-1260, 1997.

[25] D. A. S. Rees, E. Magyari, and B. Keller, "The development of the asymptotic viscous dissipation profile in a vertical free convective boundary layer flow in a porous medium", Transport in Porous Media, vol. 53, pp. 347-355, 2003

[26] D. A. Nield, "The modeling of viscous dissipation in a saturated porous medium", Journal of Heat Transfer, vol. 129 / 1459, 2007.

[27] A. Ramiar, and A. A. Ranjbar, "Effects of viscous dissipation and variable properties on nanofluids flowing two dimensional microchannels", IJE Transactions A: Basics., vol. 24, pp. 131- 142, 2011.

[28] M. J. Uddin, W. A. Khan, and A. I. Ismail, "Effect of dissipation on free convective flow of a non-Newtonian nanofluid in a porous medium with gyrotactic microorganisms", Proc IMechE Part $N: J$ Nanoengineering and Nanosystems, vol. 98, pp. 553-564, 2013.

[29] T. G. Motsumi, and O. D. Makinde, "Effects of thermal radiation and viscous dissipation on boundary layer flow of nanofluids over a permeable moving flat plate", Physica Scripta, vol. 86, no. 4, 2012.

[30] P. K. Kameswaran, M. Narayana, P. Sibanda, and P. V. S. N. Murthy, "Hydro-magnetic nanofluid flow due to a stretching or shrinking sheet with viscous dissipation and chemical reaction effects", International Journal of Heat and Mass Transfer, vol. 55, pp. 7587-7595, 2012.

[31] P. V. S. N. Murthy, A. Sutradhar, and Ch. RamReddy, "Doublediffusive free convection flow past an inclined plate embedded in a non-Darcy porous medium saturated with a Nanofluid", Transport in Porous Media, vol. 98, pp. 553-564, 2013.

[32] F. G. Blottner, "Finite-difference methods of solution of the boundary layer equations", AIAA. J., vol. 8, pp. 193-205, 1970.

[33] O. A. Plumb, and J. C. Huenefeld, "Non-Darcy natural convection from heated surfaces in saturated porous media", International Journal of Heat Mass Transfer, vol. 24, pp. 765-768, 1981 